\title{
Economic Burden Associated with Receiving Inhaled Corticosteroids with Leukotriene Receptor Antagonists or Long-Acting Beta Agonists as Combination Therapy in Older Adults
}

\author{
Shoroq M. Altawalbeh, PharmD, PhD; Carolyn T. Thorpe, PhD, MPH; Janice C. Zgibor, PhD, MPH; \\ Sandra Kane-Gill, PharmD, MS; Yihuang Kang, PhD; and Joshua M. Thorpe, PhD, MPH
}

\begin{abstract}
BACKGROUND: There is a paucity of literature on the health care expenditures associated with different pharmacologic treatments in older adults with asthma that is not well controlled on inhaled corticosteroids (ICS).

OBJECTIVE: To compare asthma-related and all-cause health care expenditures associated with leukotriene receptor antagonists (LTRA) versus longacting beta agonists (LABA) when added to ICS in older adults with asthma.

METHODS: A retrospective cohort was constructed using 2009-2010 Medicare fee-for-service medical and pharmacy claims from a $10 \%$ random sample of beneficiaries continuously enrolled in Parts A, B, and D in 2009. The sample comprised patients who were aged 65 years and older, diagnosed with asthma, and treated exclusively with ICS + LABA or ICS + LTRA. Outcomes assessed were asthma-related expenditures (medical, pharmacy, and total) and all-cause health care expenditures (medical, pharmacy, and total). Outcomes were measured from the date of the first prescription for the add-on treatment (LABA or LTRA in combination with ICS) after having at least a 4-month "wash-in" period in which patients were receiving no controller, ICS alone, or ICS plus the add-on treatment of the follow-up period. Patients were followed until death, switching to or adding the other add-on treatment, or the end of the study (December 31, 2010). Multivariable regression models with nonparametric bootstrapped standard errors were used to compare all-cause and asthma-related expenditures per patient per month (PPPM) between ICS + LABA and ICS + LTRA users. All models were adjusted for demographics, comorbidities, and countylevel health care access variables.
\end{abstract}

RESULTS: The primary analysis included 14,702 patients, of whom 12,940 were treated with ICS + LABA and 1,762 were treated with ICS + LTRA. The mean (SD) follow-up periods were $12.3( \pm 5.7)$ months for the ICS + LABA group and $15.3( \pm 5.1)$ months for the ICS + LTRA group. Adjusted asthmarelated expenditures PPPM were $\$ 400$ for the ICS + LTRA group compared with $\$ 286$ for the ICS + LABA group $(P<0.001)$. However, adjusted total allcause expenditure PPPM was significantly lower for patients treated with ICS + LTRA (\$6,087 for ICS + LTRA compared with \$6,975 for ICS + LABA, $P=0.029$ ).

CONCLUSIONS: OIder adults with asthma often experience economic burden from asthma and other chronic illnesses. Compared with ICS + LTRA, ICS + LABA was associated with lower asthma-related expenditures but with higher all-cause expenditures in older adults.

J Manag Care Spec Pharm. 2018;24(5):478-86

Copyright $\odot 2018$, Academy of Managed Care Pharmacy. All rights reserved.

\section{What is already known about this subject}

Asthma in older adults is more costly than asthma in younger adults, with higher health care utilization and expenditures Asthma-related costs for younger adults treated with inhaled corticosteroids (ICS) + long-acting beta agonists (LABA) are lower compared with those treated with ICS+leukotriene receptor antagonists (LTRA)

\section{What this study adds}

This study provides recent estimates for expenditures incurred by older adults with persistent asthma, particularly those using asthma controller medications.

Although patients treated with ICS + LABA incurred higher all-cause health expenditures than older adults treated with ICS + LTRA, they also had lower asthma-related expenditures.

A sthma places a considerable burden on the U.S. health care system, in terms of health services utilization and costs. In 2007, it was estimated that 1.75 million emergency department (ED) visits and 456,000 hospitalizations were related to asthma. ${ }^{1}$ In addition, the total annual medical cost for asthma in adults was estimated to be approximately $\$ 18$ billion. $^{2}$

Asthma in older adults is more costly than asthma in younger adults, with higher health care utilization and worse outcomes. ${ }^{3-9}$ In comparison with younger adults with asthma, older adults experience higher rates of hospital admission, longer hospital stays, higher hospital charges, higher number of prescribed medications, and higher number of ED visits. ${ }^{7,9,10}$ Few studies have estimated the direct cost of asthma in patients aged 65 years and older. ${ }^{7}$ A study conducted in 1994-1995 showed that asthma-related total direct costs were double for older adults compared with younger patients (average per year in 1994 U.S. dollars were \$1,490 vs. \$773, respectively). ${ }^{7}$ In this study, hospital inpatient care, medications, and outpatient care were found to account for 54\%, 35\%, and $7 \%$ of direct costs in older adults, respectively. ${ }^{7}$ This study did not evaluate all-cause costs for asthmatic older patients, which is a notable limitation, since the adverse effects from 


\section{Economic Burden Associated with Receiving Inhaled Corticosteroids with Leukotriene Receptor Antagonists or Long-Acting Beta Agonists as Combination Therapy in Older Adults}

pharmacological treatment can affect other comorbid conditions in this susceptible population. Also, as practices change with time, it is valuable to know how more current treatment costs compare with traditional treatment.

Long-acting beta agonists (LABA) and leukotriene receptor antagonists (LTRA) are the common add-on treatments recommended when asthma symptoms are not well controlled with low-dose inhaled corticosteroids (ICS). Current guidelines recommend LABA as a first-line add-on treatment, but evidence of its safety and effectiveness in older adults is lacking, and findings are mixed. ${ }^{11}$ Evidence from younger populations suggest that asthma-related costs for those treated with ICS + LABA were significantly lower compared with ICS + LTRA. ${ }^{12,13}$

There is a dearth of recent estimates for asthma-related and all-cause costs incurred in older adult populations. Also, up-to-date, comparative costs associated with the different add-on therapy choices have not been studied in older adult populations with asthma. Our study was designed to address these gaps in the literature by evaluating asthma-related and all-cause costs associated with the most common add-on treatments in older adult patients with persistent asthma. This investigation is intended to provide specific data on older adults that can be used for further cost-effectiveness evaluations and, ultimately, to support guidelines and protocols for asthmatic elderly care.

\section{Methods}

\section{Data Source}

The data source for this study consisted of Medicare claims and enrollment data, including 2009-2010 fee-for-service (FFS) Parts A and B medical claims, Part D prescription drug event data, and Beneficiary Summary Files (BSF) obtained from the Centers for Medicare \& Medicaid Services (CMS) for a $10 \%$ random sample of beneficiaries continuously enrolled in Parts A, B, and D in 2009.

\section{Sample}

The study population consisted of eligible Medicare beneficiaries continuously enrolled in FFS Medicare Parts A and B with Part D coverage from January 1, 2009, to December 31, 2010. To be eligible for this study, beneficiaries had to be a U.S. resident; had to be aged 65 years or more as of January 1, 2009; and had to have met criteria for having an asthma diagnosis as of January 1, 2009, as per the CMS Chronic Conditions Data Warehouse's algorithm for detecting asthma in FFS Medicare beneficiaries. ${ }^{14,15}$ According to this algorithm, asthma diagnoses were identified by at least 1 inpatient, skilled nursing facility, or home health agency claim or 2 hospital outpatient or carrier claims defined by the International Classification of Diseases, Ninth Revision, Clinical Modification (ICD-9-CM) codes for asthma (493.xx) during the year before the beginning of the study period (2008). Patients were included only if they were treated exclusively with ICS + LABA or ICS + LTRA between 2009-2010. Patients were not required to be new users of the add-on medication (LABA or LTRA), but they were required to have at least a 4-month "wash-in" period (during the study period), after which the first ICS add-on prescription was considered as the index prescription. During this wash-in period, the patient should have not received the other add-on treatment (LABA if the index prescription was ICS + LTRA or LTRA if the index prescription was ICS + LABA).

\section{Study Design}

In this retrospective national cohort study, medical and drug claims data were used to compare all-cause and asthma-related expenditures among older adults using ICS + LABA versus ICS + LTRA in an exclusive manner. Follow-up periods were started on the date of the first prescription (the index date) of the add-on treatment after the 4-month wash-in period and ended by death, switching to or adding the other add-on treatment, or the end of the study (December 31, 2010). All patient prescriptions were followed to detect any switching between LABA and LTRA add-on treatments or if being used together (switching or adding the other add-on treatment).

\section{Outcomes}

Total all-cause expenditures were calculated as the sum of total all-cause medical and total all-cause pharmacy expenditures. All-cause medical expenditures were defined as the total of Medicare payments associated with inpatient, outpatient (institutional and noninstitutional), skilled nursing facility, hospice care, home health agency, and durable medical equipment for any reason. All-cause pharmacy expenditures were defined as the sum of all expenditures associated with prescription medications in the Part D events file.

Total asthma-related expenditures were calculated as the sum of asthma-related medical and pharmacy expenditures. Asthma-related medical expenditures included Medicare payments associated with inpatient admissions, ED visits, and outpatient physician visits assigned a primary or secondary ICD-9-CM code for asthma (493.xx). Other expenditures, such as medical equipment and hospice, home health agency, and nursing home care were not included because asthma-related expenditures could not be separated from other health care expenditures for these categories (inadequate specificity). ${ }^{16}$

Asthma-related pharmacy expenditures were defined as the expenditures associated with asthma prescription medications including LABA, ICS, ICS + LABA, LTRA, oral corticosteroids or antibiotics used to treat asthma exacerbations, short-acting beta agonists, and other less commonly used asthma medications, such as methylxanthines. Asthma medications were identified using National Drug Code numbers (provided by the Healthcare Effectiveness Data and Information Set). Antibiotics and oral corticosteroids were considered to be related to 


\section{Economic Burden Associated with Receiving Inhaled Corticosteroids with Leukotriene Receptor Antagonists or Long-Acting Beta Agonists as Combination Therapy in Older Adults}

\section{TABLE 1 Study Population Characteristics for Total Sample and Asthma Add-on Medication Groups}

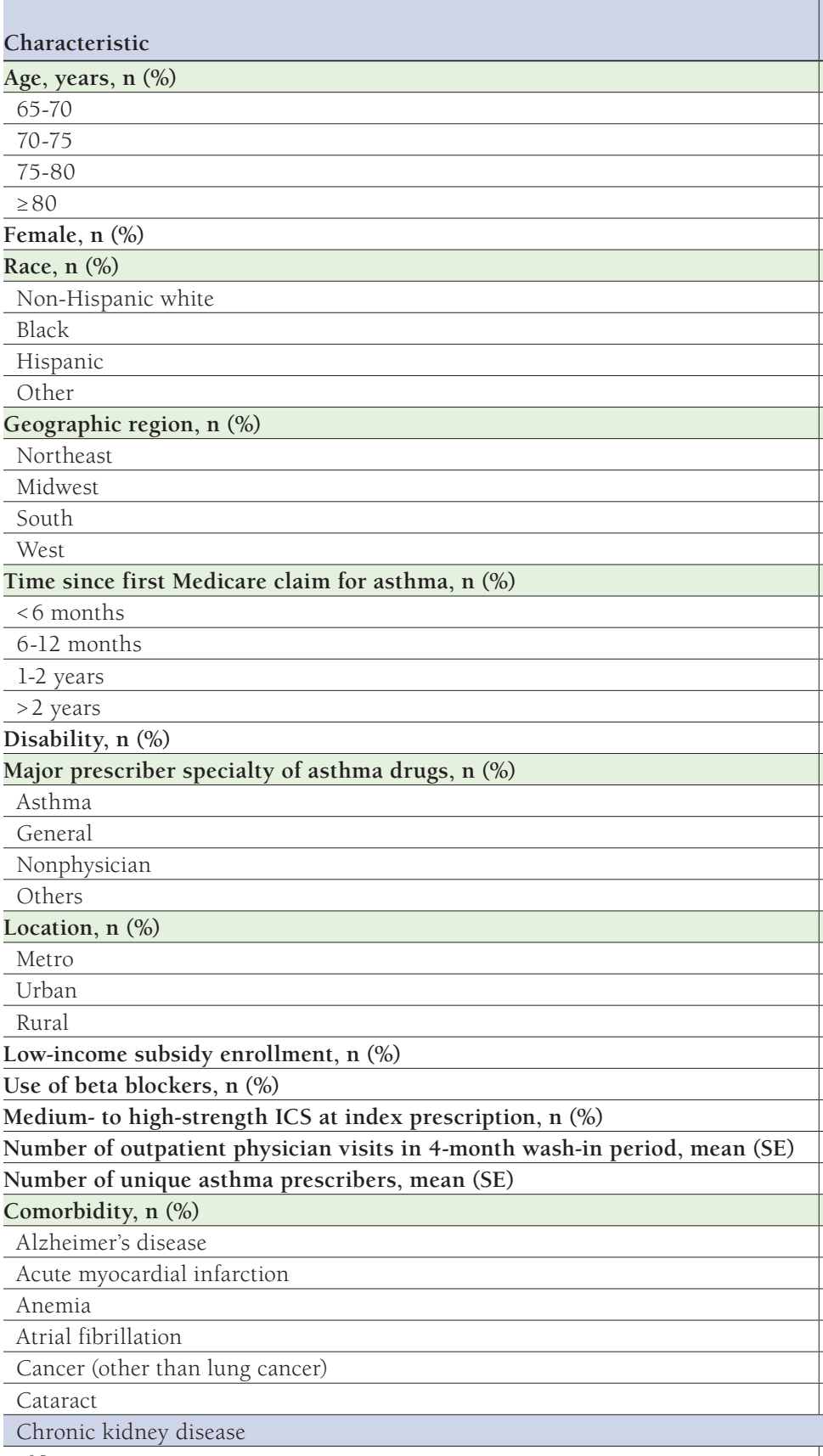

\section{2,638}

\section{0,468}

No end-stage renal disease

End-stage renal disease

Chronic obstructive pulmonary disease

Congestive heart failure

Depression

Diabetes mellitus

Glaucoma

Hip fracture

Hyperlipidemia

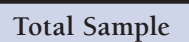

$\mathrm{N}=14,702$

2,883

3,967

3,478

4,374

10,614

11,423

1,293

1,234

738

3,192

3,31

5,32

2,868

78

944

1,682

11,295

2,495

2,893

9,360

1,019

1,430

11,471

2,71

404

6,718

5,863

11,22

4.7

1

1,068

9,974

2,990

2,260

11,440

\begin{tabular}{|c|c|}
\hline \multicolumn{2}{|c|}{$\begin{array}{l}\text { LABA Add-on } \\
\mathrm{n}=12,940\end{array}$} \\
\hline 2,495 & (19.3) \\
\hline 3,472 & (26.8) \\
\hline 3,097 & (23.9) \\
\hline 3,876 & (30.0) \\
\hline 9,225 & (71.3) \\
\hline 10,086 & $(78.0)$ \\
\hline 1,148 & (8.9) \\
\hline 1,083 & (8.4) \\
\hline 610 & $(4.7)$ \\
\hline 2,748 & $(21.2)$ \\
\hline 2,910 & (22.5) \\
\hline 4,728 & (36.5) \\
\hline 2,553 & (19.7) \\
\hline 726 & (5.6) \\
\hline 854 & (6.6) \\
\hline 1,496 & (11.6) \\
\hline 9,864 & $(76.2)$ \\
\hline 2,212 & (17.1) \\
\hline 2,521 & (19.5) \\
\hline 8,230 & (63.6) \\
\hline 907 & $(7.0)$ \\
\hline 1,282 & (9.9) \\
\hline 10,108 & (78.7) \\
\hline 2,386 & (18.6) \\
\hline 354 & (2.8) \\
\hline 5,833 & $(45.1)$ \\
\hline 5,265 & $(40.7)$ \\
\hline 10,114 & $(78.2)$ \\
\hline 4.72 & $(0.04)$ \\
\hline 1.4 & $(0.01)$ \\
\hline 2,321 & (17.9) \\
\hline 1,001 & $(7.7)$ \\
\hline 8,848 & (68.4) \\
\hline 2,688 & (20.8) \\
\hline 2,023 & (15.6) \\
\hline 10,052 & $(77.7)$ \\
\hline
\end{tabular}

LTRA Add-on $\mathrm{n}=1,762$

$388 \quad(22.0)$ $495 \quad(28.1)$ $381 \quad(21.6)$ $498 \quad(28.3)$

$1,389 \quad(78.8)$

$1,337 \quad(75.9)$

$145 \quad(8.2)$

$151 \quad$ (8.6)

128

(7.3)

$444 \quad(25.2)$

$407 \quad(23.1)$

$595 \quad(33.8)$

315

(19.1)

(5.3)

(6.4)

55

(3.1)

90
186

(5.1)

186

(10.6)

(11.4)

(76.8)

1,431

(16.1)

P Value 0.008

283

$372 \quad(21.1)$

$1,130 \quad(64.1)$

$112 \quad(6.4)$

$148 \quad(8.4)$

$1,363 \quad(78.2)$

$329 \quad(18.9)$

$50 \quad(2.9)$

$885 \quad(50.1)$

$598 \quad(33.9)$

$1,109 \quad(62.9)$

$4.42 \quad(0.10)$

\begin{tabular}{ll}
$1.7 \quad(0.02)$ \\
\hline
\end{tabular}

$317 \quad(18.0)$

$67 \quad(3.8)$

$1,126 \quad(63.9)$

$302 \quad(17.1)$

$237 \quad(13.5)$

$1,388 \quad(78.8)$

$<0.001$

$<0.001$

.

0.280
0.080

(71.2)

$9,087 \quad(70.2)$

1,38

(78.4)

$4,109 \quad(28.0)$

125

$(0.85)$

$3,737 \quad(28.9)$

11,422

(77.7)

6,316

(51.4)

116

(0.9)

$\begin{array}{ll}10,210 \quad(78.9) \\ 6,783 & (52.4)\end{array}$

$6,783 \quad(52.4)$

6,683

(45.5)

5,638

5,949

3,907

(26.6)

3,434

(43.6)

(46.0)

732

12,265

(83.4)

10,813

(26.5)

(5.1)

(83.6)

\begin{tabular}{rr|r}
1,381 & $(78.4)$ & $<0.001$ \\
372 & $(21.1)$ & $<0.001$ \\
9 & $(0.5)$ & $<0.001$ \\
1,212 & $(68.8)$ & $<0.001$ \\
773 & $(43.9)$ & $<0.001$ \\
678 & $(38.5)$ & $<0.001$ \\
734 & $(41.7)$ & 0.001 \\
473 & $(26.8)$ & 0.780 \\
71 & $(4.0)$ & 0.050 \\
1,452 & $(82.4)$ & 0.220
\end{tabular}

continued on next page 


\section{Economic Burden Associated with Receiving Inhaled Corticosteroids with Leukotriene Receptor Antagonists or Long-Acting Beta Agonists as Combination Therapy in Older Adults}

TABLE 1 Study Population Characteristics for Total Sample and Asthma Add-on Medication Groups (continued)

\begin{tabular}{|c|c|c|c|c|}
\hline Characteristic & $\begin{array}{c}\text { Total Sample } \\
\mathrm{N}=14,702\end{array}$ & $\begin{array}{l}\text { LABA Add-on } \\
\mathrm{n}=12,940\end{array}$ & $\begin{array}{l}\text { LTRA Add-on } \\
\mathrm{n}=1,762\end{array}$ & $P$ Value $^{\mathrm{a}}$ \\
\hline \multicolumn{5}{|l|}{ Comorbidity, n (\%) } \\
\hline Benign prostatic hyperplasia & $(15.7)$ & 2,104 & $(11.9)$ & $<0.001$ \\
\hline Hypertension & 13,378 & 11,815 & $(88.7)$ & $<0.001$ \\
\hline Hypothyroidism & $3,978 \quad(27.1)$ & 3,523 & $(25.8)$ & 0.210 \\
\hline Ischemic heart & $10,069 \quad(68.5)$ & $(69.5)$ & $(60.7)$ & $<0.001$ \\
\hline Lung cancer & $(3.5)$ & $(3.7)$ & $(1.7)$ & $<0.001$ \\
\hline Osteoporosis & $5,002 \quad(34.0)$ & $(33.8)$ & $(35.6)$ & 0.140 \\
\hline Rheumatoid arthritis, osteoarthritis & 10,293 & $(70.3)$ & $(67.7)$ & 0.020 \\
\hline Stroke, transient ischemic attack & $(20.3)$ & $(20.8)$ & $(17.4)$ & 0.001 \\
\hline \multicolumn{5}{|c|}{ County-level access to health care variables, mean per 10,000 older adults (SE) } \\
\hline Primary care physicians & $55.7 \quad(0.21)$ & $(0.23)$ & $(0.62)$ & 0.220 \\
\hline Medical specialty physicians & $(0.54)$ & $(0.57)$ & $(1.52)$ & 0.020 \\
\hline Allergy immunology specialty & $1.0 \quad(0.01)$ & $(0.01)$ & $(0.03)$ & 0.040 \\
\hline Cardiovascular specialty & $(0.04)$ & $(0.05)$ & $(0.12)$ & 0.030 \\
\hline Pulmonary specialty & $(0.02)$ & $(0.02)$ & $(0.06)$ & 0.180 \\
\hline Emergency medicine specialty & $(0.05)$ & $(0.06)$ & $(0.14)$ & 0.300 \\
\hline Preventive medicine specialty & $(0.01)$ & $(0.01)$ & $(0.02)$ & 0.570 \\
\hline Hospitals & $(0.01)$ & $(0.01)$ & $(0.04)$ & 0.030 \\
\hline Hospitals with emergency department & $1.0 \quad(0.01)$ & $(0.01)$ & $(0.03)$ & 0.430 \\
\hline Average household size & $(0.02)$ & $(0.02)$ & $(0.01)$ & 0.004 \\
\hline Percentage below poverty & $11.4 \quad(0.04)$ & $(0.04)$ & $(0.11)$ & 0.070 \\
\hline
\end{tabular}

asthma exacerbation if claimed on the same day or within the next 7 days after face-to-face outpatient physician visits in the carrier file (identified through Current Procedural Terminology codes) that associated with a primary or secondary asthma diagnosis code. ${ }^{17}$

Direct medical (all-cause and asthma-related) health care spending was defined as the amount paid by Medicare. Medical expenditures paid for by other third-party sources were not included. Pharmacy expenditures were defined as the sum of the gross amount of pharmacy prescriptions (all-cause and asthma-related). The gross drug cost included Medicare and patient paid amounts. All asthma-related and all-cause expenditures were divided by follow-up months for each patient and reported as per-patient-per-month (PPPM) expenditures.

\section{Covariates}

In addition to the main explanatory variable (treatment group with either ICS+LABA or ICS+LTRA), we adjusted for a range of baseline patient characteristics in multivariate models, including demographics, comorbidities, and county-level health access variables. The BSF was used to extract information regarding patient age (as of January 1, 2009), patient race/ ethnicity (non-Hispanic white, black, Hispanic, or others), region of country (Northeast, Midwest, South, West), sex, enrollment in the low-income subsidy, disability indicator, asthma duration since the first diagnosis in Medicare data, and several comorbidities. The BSF includes diagnosis indicators for 21 chronic conditions along with the dates for their first diagnoses in Medicare data. In order to be considered as preexisting conditions, the first diagnoses in Medicare data were required to be before the beginning date of patient follow-up.

Other covariates included in the model were use of beta blockers, since these medications may affect asthma outcomes $^{18}$; ICS strength at the date of starting follow-up as a proxy for ICS dose and asthma severity; outpatient doctor visits during the wash-in period; and prescriber characteristics, including number of unique prescribers of asthma medications, as well as the specialty of the prescriber who accounted for the greatest number of asthma prescriptions.

Finally, Medicare data were linked to the Health Resource and Services Administration's Area Resource File to extract county-level health care access variables, including number of primary care physicians, medical specialty physicians, allergy immunology specialty, cardiovascular specialty, pulmonary specialty, emergency medicine specialty, preventive medicine specialty, hospital number, and hospitals with EDs. These variables were calculated as the number per 10,000 older adult residents for each county. County-level rurality/urbanicity, average household size, and percentage below poverty were also included. 
TABLE 2 Primary Analysis: All-Cause and Asthma-Related Expenditures PPPM by Asthma Treatment Group for Medicare Recipients from 2009-2010

\begin{tabular}{|c|c|c|c|c|c|}
\hline Unadjusted Expenditures, \$ & $\begin{array}{c}\text { Total Sample } \\
\mathrm{N}=14,702 \\
\text { Mean }(95 \% \mathrm{CI})\end{array}$ & $\begin{array}{l}\text { LABA Add-on } \\
\text { Group } \\
\mathrm{n}=12,940 \\
\text { Mean }(95 \% \mathrm{CI})\end{array}$ & $\begin{array}{l}\text { LTRA Add-on } \\
\text { Group } \\
\mathbf{n}=1,762 \\
\text { Mean }(95 \% \mathrm{CI})\end{array}$ & $P$ Value ${ }^{a}$ & Favor \\
\hline Total all-cause expenditures & $\begin{array}{c}6,868 \\
(6,392-7,344)\end{array}$ & $\begin{array}{c}7,122 \\
(6,588-7,655)\end{array}$ & $\begin{array}{c}5,009 \\
(4,356-5,661)\end{array}$ & 0.005 & LTRA \\
\hline All-cause medical expenditures & $\begin{array}{c}6,377 \\
(5,903-6,851)\end{array}$ & $\begin{array}{c}6,641 \\
(6,110-7,172)\end{array}$ & $\begin{array}{c}4,437 \\
(3,787-5,087) \\
\end{array}$ & 0.003 & LTRA \\
\hline All-cause pharmacy expenditures & $\begin{array}{c}491 \\
(480-503)\end{array}$ & $\begin{array}{c}481 \\
(468-493)\end{array}$ & $\begin{array}{c}572 \\
(541-602)\end{array}$ & $<0.001$ & LABA \\
\hline Total asthma-related expenditures & $\begin{array}{c}299 \\
(284-315) \\
\end{array}$ & $\begin{array}{c}286 \\
(269-303) \\
\end{array}$ & $\begin{array}{c}395 \\
(363-428) \\
\end{array}$ & $<0.001$ & LABA \\
\hline Asthma-related medical expenditures ${ }^{b}$ & $\begin{array}{c}157 \\
(142-171) \\
\end{array}$ & $\begin{array}{c}152 \\
(136-167) \\
\end{array}$ & $\begin{array}{c}193 \\
(164-223)\end{array}$ & 0.060 & Neither \\
\hline Asthma-related pharmacy expenditures & $\begin{array}{c}143 \\
(137-149)\end{array}$ & $\begin{array}{c}135 \\
(129-141)\end{array}$ & $\begin{array}{c}202 \\
(186-218) \\
\end{array}$ & $<0.001$ & LABA \\
\hline Adjusted Expenditures, \$ & $\begin{array}{l}\text { LABA Add-On } \\
\text { Group } \\
\mathrm{n}=12,940 \\
\text { Mean }(95 \% \mathrm{CI})\end{array}$ & $\begin{array}{l}\text { LTRA Add-On } \\
\text { Group } \\
\mathbf{n}=1,762 \\
\text { Mean }(95 \% \mathrm{CI})\end{array}$ & $\begin{array}{l}\text { Difference in } \\
\text { Expenditures }\end{array}$ & $P$ Valuec & Favor \\
\hline Total all-cause expenditures & $\begin{array}{c}6,975 \\
(6,489-7,460) \\
\end{array}$ & $\begin{array}{c}6,087 \\
(5,460-6,715)\end{array}$ & 888 & 0.029 & LTRA \\
\hline All-cause medical expenditures & $\begin{array}{c}6,496 \\
(6,006-6,986) \\
\end{array}$ & $\begin{array}{c}5,501 \\
(4,893-6,108) \\
\end{array}$ & 995 & 0.012 & LTRA \\
\hline All-cause pharmacy expenditures & $\begin{array}{c}478 \\
(466-491) \\
\end{array}$ & $\begin{array}{c}587 \\
(556-618) \\
\end{array}$ & 109 & $<0.001$ & LABA \\
\hline Total asthma-related expenditures & $\begin{array}{c}286 \\
(270-301)\end{array}$ & $\begin{array}{c}400 \\
(364-436)\end{array}$ & 114 & $<0.001$ & LABA \\
\hline Asthma-related medical expenditures ${ }^{\mathrm{b}}$ & $\begin{array}{c}151 \\
(137-165) \\
\end{array}$ & $\begin{array}{c}199 \\
(167-231) \\
\end{array}$ & 48 & 0.002 & LABA \\
\hline Asthma-related pharmacy expenditures & $\begin{array}{c}135 \\
(129-141) \\
\end{array}$ & $\begin{array}{c}201 \\
(184-217) \\
\end{array}$ & 66 & $<0.001$ & LABA \\
\hline \multicolumn{6}{|c|}{$\begin{array}{l}\text { aExpenditures were compared using the Wilcoxon test. } \\
\text { bAsthma-related medical expenditures included inpatient, ED, and outpatient physician visit expenditures. } \\
\text { ¿Expenditures were compared using nonparametric bootstrap procedures with 1,000 replications. } \\
C I=\text { confidence interval; } E D=\text { emergency department; } L A B A=\text { long acting beta agonist; LTRA=leukotriene receptor antagonist; PPPM=per patient per month. }\end{array}$} \\
\hline
\end{tabular}

\section{Statistical Analysis}

Primary Analysis. All analyses were conducted in Stata version 13 (StataCorp, College Station, TX). Bivariate analyses (two-sample t-tests and Wald chi-square tests) were used to compare differences in population characteristics between the 2 treatment groups. Unadjusted PPPM expenditures were summarized using descriptive statistics for the total sample and by treatment group (ICS + LABA vs. ICS + LTRA). Group comparisons of expenditures were tested by the Wilcoxon test.

Nonparametric bootstrap procedures with 1,000 replications were used to compare health care expenditures and asthma-related expenditures between the 2 groups. Bootstrapping is frequently used when evaluating differences in expenditures. ${ }^{19-21}$ Bootstrapping compares arithmetic means without making assumptions about the distribution. . $22,23^{2}$ Further, the nonparametric bootstrapping method is shown to be robust for data with a larger proportion with no health care costs. ${ }^{24}$ Six bootstrapping regression models were conducted in which outcomes were total all-cause expenditures PPPM, total all-cause medical expenditures PPPM, total all-cause pharmacy expenditures PPPM, total asthma-related expenditures PPPM, asthma-related medical expenditures PPPM, and asthmarelated pharmacy expenditures PPPM.

Treatment group was the main explanatory variable in all models, adjusting for all other covariates as previously described. Adjusted predicted expenditures PPPM (or marginal expenditures) were predicted after regression models using the mean values for the other independent variables.

Sensitivity Analysis: Discontinuation Design. In this analysis, patient prescription fills were followed to exclude periods of no treatment supply based on the days supply field in the Medicare Part D event file. As an indicator of discontinuation, patients were censored once they had more than 30 days without a treatment supply for either the ICS or the add-on treatment. 


\begin{tabular}{|c|c|c|c|c|c|}
\hline Adjusted Expenditures & $\begin{array}{l}\text { LABA Add-On } \\
\text { Group, } \$ \\
\mathrm{n}=12,868 \\
\text { Mean }(95 \% \mathrm{CI})\end{array}$ & $\begin{array}{l}\text { LTRA Add-On } \\
\quad \text { Group, } \$ \\
\mathbf{n}=1,498 \\
\text { Mean }(95 \% \mathrm{CI})\end{array}$ & $\begin{array}{l}\text { Difference in } \\
\text { Expenditures }\end{array}$ & $P$ Value ${ }^{a}$ & Favor \\
\hline Total all-cause expenditures & $\begin{array}{c}6,797 \\
(6,090-7,504) \\
\end{array}$ & $\begin{array}{c}6,919 \\
(5,659-8,180)\end{array}$ & & 0.869 & Neither \\
\hline All-cause medical expenditures & $\begin{array}{c}6,260 \\
(5,550-6,970)\end{array}$ & $\begin{array}{c}6,139 \\
(4,809-7,469)\end{array}$ & & 0.875 & Neither \\
\hline All-cause pharmacy expenditures & $\begin{array}{c}537 \\
(525-550) \\
\end{array}$ & $\begin{array}{c}780 \\
(727-834) \\
\end{array}$ & 243 & $<0.001$ & LABA \\
\hline Total asthma-related expenditures & $\begin{array}{c}331 \\
(312-350)\end{array}$ & $\begin{array}{c}507 \\
(441-573)\end{array}$ & 176 & $<0.001$ & LABA \\
\hline Asthma-related medical expenditures ${ }^{b}$ & $\begin{array}{c}150 \\
(132-169)\end{array}$ & $\begin{array}{c}184 \\
(128-241)\end{array}$ & & 0.240 & Neither \\
\hline Asthma-related pharmacy expenditures & $\begin{array}{c}180 \\
(174-187)\end{array}$ & $\begin{array}{c}323 \\
(289-357) \\
\end{array}$ & 143 & $<0.001$ & LABA \\
\hline \multicolumn{6}{|c|}{$\begin{array}{l}\text { aExpenditures were compared using nonparametric bootstrap procedures with 1,000 replications. } \\
\text { basthma-related medical expenditures included inpatient, ED, and outpatient physician visit expenditures. } \\
C I=\text { confidence interval; } E D=\text { emergency department; } L A B A=\text { long acting beta agonist; LTRA=leukotriene receptor antagonist; PPPM = per patient per month. }\end{array}$} \\
\hline
\end{tabular}

\section{Results}

\section{Patient Characteristics and Baseline Comparisons}

A total of 14,702 patients met the inclusion criteria, of whom 1,762 were receiving ICS+LTRA and 12,940 were receiving ICS + LABA in an exclusive manner. The average follow-up periods (standard deviation $[S D])$ were $12.3( \pm 5.7)$ months for the ICS + LABA group (median $=12.9$ months $)$ and $15.3( \pm 5.1)$ months for the ICS + LTRA group (median= 17.6 months). The mean age of the study cohort was 76.5 years; $72 \%$ were female; and $78 \%$ were non-Hispanic whites. The most common comorbid conditions were hypertension (91\%), hyperlipidemia (83\%), cataracts $(77.8 \%)$ and chronic obstructive pulmonary disease (77.7\%). The average number of comorbid chronic diseases per patient was 9 overall-9 for the ICS + LABA group compared with 8 for the ICS+LTRA group. The characteristics of the study cohort are summarized in Table 1.

\section{Health Care Expenditures}

Unadjusted Expenditures. Average unadjusted all-cause and asthma-related expenditures PPPM are summarized in Table 2. The average total expenditures PPPM were lower for patients treated with ICS + LTRA compared with those treated with ICS + LABA ( $\$ 5,009$ vs. $\$ 7,122$, respectively); however, asthmarelated expenditures PPPM were higher for patients treated with ICS + LTRA compared with those treated with ICS + LABA (\$395 vs. \$286). Similarly, ICS+LTRA treatment was associated with lower all-cause medical expenditures but with indifferent asthmarelated medical expenditures compared with the ICS + LABA group. In contrast, all-cause and asthma-related pharmacy expenditures were higher for patients treated with ICS+LTRA. All comparisons were statistically significant $(P<0.001)$.
Adjusted Regression Models. Adjusted predicted all-cause and asthma-related expenditures PPPM are summarized in Table 2.

All-cause expenditures: All-cause total and medical expenditures PPPM were significantly lower in patients treated with ICS + LTRA versus those treated with ICS + LABA. Total allcause expenditures PPPM were $\$ 6,975$ for the ICS+LABA group and $\$ 6,087$ for the ICS+LTRA group $(P=0.029)$. The corresponding values for total medical expenditures PPPM were $\$ 6,496$ for the ICS + LABA group compared with $\$ 5,501$ for the ICS+LTRA group $(P=0.012)$. All-cause pharmacy expenditures PPPM were significantly higher for patients treated with ICS + LTRA (\$587) compared with those treated with ICS + LABA $(\$ 478, P<0.001)$.

Asthma-related expenditures: Compared with ICS + LTRA, treatment with ICS + LABA was associated with significantly lower total asthma-related expenditures, asthma-related medical expenditures, and asthma-related pharmacy expenditures (differences of $\$ 114, \$ 48$ and $\$ 66$ PPPM, respectively).

Sensitivity Analysis: Discontinuation Design. After applying the discontinuation design, the average follow-up periods (SD) were $3.9( \pm 3.7)$ months for the ICS + LABA group (median = 1.9 months $)$ and $4.6( \pm 5.1)$ months for the ICS + LTRA group (median $=2.3$ months). Following patients for periods of continuous medication supply showed that the ICS + LABA group was associated with similar all-cause total and medical expenditures PPPM but with significantly lower all-cause pharmacy expenditures PPPM compared with the ICS+LTRA group. Also, ICS + LABA treatment was associated with significantly lower total asthma-related and pharmacy expenditures PPPM; 
however, asthma-related medical expenditures PPPM (inpatient, ED, and outpatient visits) were not different between 2 groups (Table 3 ).

\section{Discussion}

This economic evaluation showed that older adult patients treated with ICS + LABA incurred significantly lower asthmarelated expenditures compared with those treated with ICS + LTRA. However, the total and medical all-cause expenditures incurred were lower for patients treated with ICS + LTRA. The difference in total and medical all-cause expenditures, however, turned out to be insignificant after following patients for periods of continuous medication supply. Economic evaluations of younger populations have consistently demonstrated that patients receiving ICS + LTRA incur higher asthma-related costs compared with patients receiving ICS + LABA..$^{12,13,25}$ In a retrospective observational study, Tan et al. (2009) showed that the total asthma-related annual costs in the ICS + LTRA group was 38\% higher than those in the ICS + LABA group $(P<0.001){ }^{13}$ Similarly, Lee et al. (2010) reported higher adjusted mean total asthma-related costs in patients treated with ICS + LTRA compared with ICS + LABA patients ( $\$ 1,223$ vs. $\$ 873$ per year). ${ }^{12}$ In agreement with these findings, our study showed that ICS+LTRA patients incurred higher asthma-related medical and prescription expenditures in the older adult population. These results are consistent with previous findings in older adult populations, which have shown that ICS + LABA treatment is associated with lower likelihood of asthma exacerbations. ${ }^{11}$

Pharmacy spending was a major driver of asthma expenditures and was significantly higher in patients treated with ICS + LTRA (\$201 PPPM) compared with patients treated with ICS + LABA (\$135 PPPM). Our results agree with previous evidence from younger ages in which asthma drugs cost more for patients treated with ICS + LTRA than for patients treated with ICS + LABA ( $\$ 987$ vs. \$606, respectively). ${ }^{25}$ Although our conclusion was similar to those reported for younger ages, asthma expenditures estimates in older adults were much higher compared with those estimated in younger adults or age-pooled research. ${ }^{12,13,25,26}$

Few studies have evaluated all-cause costs in asthmatic adults. Stempel et al. (2002) found that nonelderly patients treated with ICS + LABA incurred significantly lower total all-cause costs compared with those treated with ICS + LTRA $\left(\$ 3,466\right.$ ICS+LABA vs. $\$ 4,346$ ICS+LTRA per year). ${ }^{25}$ In comparison, our investigation showed that total all-cause expenditures were lower in patients treated with ICS + LTRA compared with those treated with ICS+LABA $(\$ 6,975$ in ICS + LABA group and \$6,087 in ICS + LTRA group). This finding suggests that the higher asthma expenditures associated with ICS + LTRA were offset by lower expenditures from other conditions in older adults. Older asthmatic patients treated with ICS + LABA have been shown to have more cardiovascular hospitalization or ED visits compared with patients treated with ICS+ LTRA. ${ }^{11}$

Unsurprisingly, total all-cause estimates were much higher than their corresponding values estimated from younger ages. ${ }^{16,25}$ Such high numbers are likely because of the high prevalence of multimorbidity among older adults noted in this study. The average number of comorbid conditions across the whole sample was 9 chronic diseases per patient. A CMS investigation that analyzed differences in Medicare FFS use (Medicare perspective) by beneficiary characteristics including chronic conditions found an influential effect of chronic conditions on Medicare payments. ${ }^{27}$ The average annual payments for beneficiaries with 9 comorbid conditions were $\$ 57,806$ for Part A and \$22,404 for Part B in 2010 compared with $\$ 10,989$ and $\$ 9,189$, respectively, for beneficiaries with 4 comorbid conditions.

Finally, this study showed that all-cause prescription drug expenditures were significantly higher in patients treated with ICS+LTRA compared with those treated with ICS + LABA. This finding was driven, in part, by asthma treatments comprising approximately $28 \%$ of total drug expenditures in the ICS + LABA group and 34\% of total drug expenditures in the ICS + LTRA group.

\section{Limitations}

This study has several limitations. Expenditures were measured from the Medicare perspective, and patient out-of-pocket and third-party expenditures were not included. Hence, this study might have underestimated the societal spending of asthma. However, in 2014, Medicare expenditures contributed to $20 \%$ of all U.S. health.care expenditures. ${ }^{28}$ In asthma-related expenses, only inpatient, ED, and outpatient physician visits and drugs were included; however, aside from medication expenditures, exacerbations resulting in hospitalizations or ED visits were shown to have the greatest economic effect among all sources of direct costs. ${ }^{12,13,29}$ We may not have adequately captured differences in asthma severity in the ICS + LABA versus ICS+LTRA groups; however, in our analysis, we have adjusted for ICS strength as a proxy for asthma severity.

Because we used the prevalent users design in which incident and prevalent users were included regardless of the initiation time, we could not account for baseline all-cause and asthma-related costs. However, our design enabled larger sample size, enhanced generalizability, and allowed for the evaluation of long-term treatment effects. Finally, although we did account for a wide variety of covariables and potential confounders, the possibility of residual selection bias cannot be ruled out completely in observational study designs. 


\section{Economic Burden Associated with Receiving Inhaled Corticosteroids with Leukotriene Receptor Antagonists or Long-Acting Beta Agonists as Combination Therapy in Older Adults}

\section{Conclusions}

Overall, all-cause and asthma-related per-patient direct expenditures for asthmatic FFS Medicare beneficiaries were substantial. ICS + LTRA treatment was associated with higher asthma-related expenditures compared with ICS + LABA treatment; however, all-cause expenditures were higher for patients treated with ICS + LABA, except for prescription expenditures. All-cause total and medical expenditures were not different between the 2 groups after following patients only for periods of continuous medication supply.

\section{Authors}

SHOROQ M. ALTAWALBEH, PharmD, PhD, Department of Clinical Pharmacy, Jordan University of Science and Technology School of Pharmacy, Irbid, Jordan, and Department of Pharmacy and Therapeutics, University of Pittsburgh School of Pharmacy, Pittsburgh, Pennsylvania. CAROLYN T. THORPE, PhD, MPH, and JOSHUA M. THORPE, PhD, MPH, Department of Pharmacy and Therapeutics, University of Pittsburgh School of Pharmacy, and Center for Health Equity Research and Promotion, Veterans Affairs Pittsburgh Healthcare System, Pittsburgh, Pennsylvania. JANICE C. ZGIBOR, PhD, MPH, Department of Epidemiology and Biostatistics, University of South Florida College of Public Health, Tampa; SANDRA KANE-GILL, PharmD, MS, Department of Pharmacy and Therapeutics, University of Pittsburgh School of Pharmacy, Pittsburgh, Pennsylvania; and YIHUANG KANG, PhD, Department of Information Management, National Sun Yat-sen University, Kaohsiung, Taiwan.

AUTHOR CORRESPONDENCE: Shoroq M. Altawalbeh, PharmD, PhD, Department of Clinical Pharmacy, Jordan University of Science and Technology School of Pharmacy, P.O. Box 3030, Irbid 22110, Jordan. Tel.: +962(0)27201000; E-mail: smaltawalbeh@just.edu.jo.

\section{DISCLOSURES}

Support for this study was provided by the University of Pittsburgh School of Pharmacy and the Pittsburgh Claude D. Pepper Older Americans Independence Center (NIA P30 AGAG024827). C. Thorpe reports grants from the National Institute of Aging during the conduct of this study. The other authors have nothing to disclose.

\section{REFERENCES}

1. American Lung Association. Trends in asthma morbidity and mortality. September 2012. Available at: http://www.lung.org/assets/documents/ research/asthma-trend-report.pdf. Accessed March 19, 2018.

2. Sullivan PW, Ghushchyan VH, Slejko JF, Belozeroff V, Globe DR, Lin SL. The burden of adult asthma in the United States: evidence from the Medical Expenditure Panel Survey. J Allergy Clin Immunol. 2011;127(2):363-69.el-3.

3. Hanania NA, King MJ, Braman SS, et al. Asthma in the elderly: current understanding and future research needs-a report of a National Institute on Aging (NIA) workshop. J Allergy Clin Immunol. 2011;128(3 Suppl):S4-24.

4. Lee KH, Chin NK, Lim TK. Asthma in the elderly-a more severe disease. Singapore Med J. 2000;41(12):579-81
5. Moorman JE, Rudd RA, Johnson CA, et al. National surveillance for asthma-United States, 1980-2004. MMWR Surveill Summ. 2007;56(8):1-54.

6. Piecoro LT, Potoski M, Talbert JC, Doherty DE. Asthma prevalence, cost, and adherence with expert guidelines on the utilization of health care services and costs in a state Medicaid population. Health Serv Res. 2001;36(2):357-71.

7. Plaza V, Serra-Batlles J, Ferrer M, Morejon E. Quality of life and economic features in elderly asthmatics. Respiration. 2000;67(1):65-70.

8. Talreja N, Baptist AP. Effect of age on asthma control: results from the National Asthma Survey. Ann Allergy Asthma Immunol. 2011;106(1):24-29.

9. Tsai CL, Lee WY, Hanania NA, Camargo CA Jr. Age-related differences in clinical outcomes for acute asthma in the United States, 2006-2008. J Allergy Clin Immunol. 2012;129(5):1252-58.e1

10. Banerji A, Clark S, Afilalo M, Blanda MP, Cydulka RK, Camargo CA Jr. Prospective multicenter study of acute asthma in younger versus older adults presenting to the emergency department. J Am Geriatr Soc. 2006;54(1):48-55.

11. Altawalbeh SM, Thorpe CT, Zgibor JC, Kane-Gill S, Kang Y, Thorpe JM. Antileukotriene agents versus long-acting beta-agonists in older adults with persistent asthma: a comparison of add-on therapies. J Am Geriatr Soc. 2016;64(8):1592-600

12. Lee TA, Chang CL, Stephenson JJ, et al. Impact of asthma controller medications on medical and economic resource utilization in adult asthma patients. Curr Med Res Opin. 2010;26(12):2851-60.

13. Tan H, Sarawate C, Singer J, et al. Impact of asthma controller medications on clinical, economic, and patient-reported outcomes. Mayo Clin Proc. 2009;84(8):675-84

14. Centers for Medicare \& Medicaid Services. Chronic Condition Data Warehouse. 2014. Accessed July 2015. Available at: https://www.ccwdata. org/web/guest/condition-categories. Accessed March 19, 2018.

15. Dombkowski KJ, Wasilevich EA, Lyon-Callo S, Nguyen TQ, Medvesky MG, Lee MA. Asthma surveillance using Medicaid administrative data: a call for a national framework. J Public Health Manag Pract. 2009;15(6):485-93.

16. Zeiger RS, Hay JW, Contreras R, et al. Asthma costs and utilization in a managed care organization. J Allergy Clin Immunol. 2008;121(4):885-92.e5.

17. Dalal AA, Christensen L, Liu F, Riedel AA. Direct costs of chronic obstructive pulmonary disease among managed care patients. Int J Chron Obstruct Pulmon Dis. 2010;5:341-49.

18. Brooks TW, Creekmore FM, Young DC, Asche CV, Oberg B, Samuelson WM. Rates of hospitalizations and emergency department visits in patients with asthma and chronic obstructive pulmonary disease taking beta-blockers. Pharmacotherapy. 2007;27(5):684-90.

19. Grieve R, Thompson S, Normand C, Suri R, Bush A, Wallis C. A cost-effectiveness analysis of rhDNase in children with cystic fibrosis. Int J Technol Assess Health Care. 2003;19(1):71-79.

20. Laliberte F, Cloutier M, Crivera C, et al. Effect of rivaroxaban versus warfarin on health care costs among nonvalvular atrial fibrillation patients: observations from rivaroxaban users and matched warfarin users. Adv Ther 2015;32(3):216-27.

21. Struijs PA, Korthals-de Bos IB, van Tulder MW, van Dijk CN, Bouter LM, Assendelft WJ. Cost effectiveness of brace, physiotherapy, or both for treatment of tennis elbow. Br J Sports Med. 2006;40(7):637-43.

22. Barber JA, Thompson SG. Analysis of cost data in randomized trials: an application of the non-parametric bootstrap. Stat Med. 2000;19(23):3219-36.

23. Briggs A, Gray A. The distribution of health care costs and their statistical analysis for economic evaluation. J Health Serv Res Policy. 1998;3(4):233-45.

24. Gilleskie DB, Mroz TA. A flexible approach for estimating the effects of covariates on health expenditures. J Health Econ. 2004;23(2):391-18.

25. Stempel DA, O'Donnell JC, Meyer JW. Inhaled corticosteroids plus salmeterol or montelukast: effects on resource utilization and costs. J Allergy Clin Immunol. 2002;109(3):433-39. 
26. Chastek B, Korrer S, Nagar SP, et al. Economic burden of illness among patients with severe asthma in a managed care setting. J Manag Care Spec Pharm. 2016;22(7):848-61. Available at: https://www.jmcp.org/doi/10.18553/ jmcp.2016.22.7.848.

27. Erdem E, Prada SI, Haffer SC. Medicare payments: how much do chronic conditions matter? Medicare Medicaid Res Rev. 2013;3(2).

28. Centers for Medicare $\&$ Medicaid Services. NHE fact sheet. 2014. Accessed July 2016. Available at: https://www.cms.gov/research-statisticsdata-and-systems/statistics-trends-and-reports/nationalhealthexpenddata/ nhe-fact-sheet.html. Accessed March 19, 2018.
29. Stanford RH, Blanchette CM, Roberts MH, Petersen H, Fuhlbrigge AL. Effect of combination fluticasone propionate and salmeterol or inhaled corticosteroids on asthma-related outcomes in a Medicare-eligible population. Am J Geriatr Pharmacother. 2012;10(6):343-51. 